\title{
Hyperglycemia and Incidence of Frailty and Lower Extremity Mobility Limitations in Older Women
}

\author{
Rita R. Kalyani, MD, MHS, * Jing Tian, MS, ${ }^{\dagger}$ Qian-Li Xue, PhD, ${ }^{\dagger \neq}$ Jeremy Walston, MD, ${ }^{\dagger *}$ \\ Anne R. Cappola, MD, ScM, ${ }^{\S}$ Linda P. Fried, MD, MPH," Frederick L. Brancati, MD, MHS, ${ }^{\prime \prime}$ and \\ Caroline S. Blaum, MD, MS**
}

OBJECTIVES: To determine the degree to which hyperglycemia predicts the development of frailty and lower extremity mobility limitations.

DESIGN: Secondary data analysis of longitudinal data collected in a prospective cohort study.

SETTING: Baltimore, Maryland.

PARTICIPANTS: Three hundred twenty-nine women from the Women's Health and Aging Study II aged 70 to 79 at baseline who had all variables needed for analysis.

MEASUREMENTS: Glycosylated hemoglobin (HbA1c) at baseline, categorized as less than $5.5 \%, 5.5 \%$ to $5.9 \%$, $6.0 \%$ to $6.4 \%, 6.5 \%$ to $7.9 \%$, and $8.0 \%$ and greater, was the independent variable. The incidence of frailty and lower extremity mobility limitations (based on selfreported walking difficulty, walking speed, and Short Performance Physical Battery score) was determined (follow-up $\approx 9$ years). Frailty was assessed using the Cardiovascular Health Study criteria. Covariates included demographic characteristics, body mass index, interleukin6 level, and clinical history of comorbidities. Statistical analyses included Kaplan-Meier survival curves and Cox regression models adjusted for important covariates.

RESULTS: In time-to-event analyses, HbA1c category was associated with incidence of walking difficulty $(P=.049)$ and low physical performance $(P=.001)$; association with incidence of frailty and low walking speed had a trend toward significance (both $P=.10$ ). In regression models adjusted for demographic characteristics, HbA1c of $8.0 \%$ or greater (vs $<5.5 \%$ ) was associated with an approxi-

From the *Divisions of Endocrinology, ${ }^{\dagger}$ Geriatric Medicine and Gerontology, ${ }^{\ddagger}$ Center on Aging and Health, Johns Hopkins University, Baltimore, Maryland; ${ }^{\S}$ Division of Endocrinology, Diabetes and

Metabolism, University of Pennsylvania, Philadelphia, Pennsylvania;

"Department of Epidemiology, Mailman School of Public Health,

Columbia University, New York, New York; "Division of General Internal

Medicine, Johns Hopkins University, Baltimore, Maryland; and

**Division of Geriatric Medicine, University of Michigan, Ann Arbor, Michigan.

Address correspondence to Rita R. Kalyani, 1830 East Monument Street, Suite 333, Baltimore, MD 21287. E-mail: rrastogi@jhmi.edu

DOI: $10.1111 / \mathrm{j} .1532-5415.2012 .04099 . \mathrm{x}$ mately three-times greater risk of incident frailty and three to five times greater risk of lower extremity mobility limitations (all $P<.05$ ). In fully adjusted models, HbA1c of $8.0 \%$ or greater (vs $<5.5 \%$ ) was associated with incident frailty (hazard ratio $(\mathrm{HR})=3.33,95 \%$ confidence interval $(\mathrm{CI})=1.24-8.93)$, walking difficulty $(\mathrm{HR}=3.47,95 \%$ $\mathrm{CI}=1.26-9.55)$, low walking speed $(\mathrm{HR}=2.82,95 \%$ $\mathrm{CI}=1.19-6.71), \quad$ and low physical performance $(\mathrm{HR}=3.60,95 \% \mathrm{CI}=1.52-8.53)$.

CONCLUSION: Hyperglycemia is associated with the development of frailty and lower extremity mobility limitations in older women. Future studies should identify mediators of these relationships. J Am Geriatr Soc 60:17011707, 2012.

Key words: hyperglycemia; elderly; frailty; mobility; disability

$\mathrm{D}$ iabetes mellitus in elderly adults is a growing public health concern, with almost two-thirds of older U.S. adults having diabetes mellitus or pre-diabetes mellitus. ${ }^{1}$ The numbers of persons with diabetes mellitus will almost double by $2030 .{ }^{2}$ In older people, diabetes mellitus can have a significant effect on physical functioning and has been associated with lower extremity mobility limitations. ${ }^{3,4}$ Diabetes mellitus has also been associated with frailty, a geriatric condition of physiological vulnerability to stressors associated with adverse outcomes such as disability and mortality, ${ }^{5-8}$ but whether hyperglycemia per se predicts the development of frailty or lower extremity mobility limitations has not been fully described.

Cross-sectional studies have previously demonstrated that hyperglycemia is associated with frailty. ${ }^{9}$ Different dynamics of glucose and insulin in response to a glucose challenge have also been reported in frail and nonfrail women, with 2-hour post-oral glucose tolerance test (OGTT) levels of glucose and insulin better discriminating frailty status than fasting values, ${ }^{8}$ although the direction of 
the association between frailty status and abnormalities in glucose metabolism remains unclear. Longitudinal associations between insulin resistance and incident frailty have been described in the Cardiovascular Health Study (CHS). ${ }^{10}$ Nonetheless, the use of short-term fasting measures of glycemia have limited these studies, whereas biomarkers such as glycosylated hemoglobin (HbA1c), which reflect exposure to glucose over the past 3 months and are influenced by fasting and postprandial hyperglycemia, may be less variable. Hyperglycemia has also been found to be cross-sectionally associated with lower extremity disability ${ }^{4,9}$ but to the knowledge of the authors of the current study, associations between hyperglycemia and declines in lower extremity mobility function over time have not been previously explored.

The goal of the present study was to describe the association between hyperglycemia and incident frailty and lower extremity mobility limitations in a longitudinal cohort of older, community-dwelling women. The hypotheses were that hyperglycemia (assessed according to $\mathrm{HbA1c}$ ) would predict the development of frailty and lower extremity limitations; that the association between hyperglycemia and frailty and lower extremity limitations would be independent of potential confounders; and that the association between hyperglycemia and frailty and lower extremity limitations would be nonlinear.

\section{METHODS}

\section{Subjects}

The study population consisted of women aged 70 to 79 at baseline enrolled in the Women's Health and Aging Study II who represented the two-thirds least disabled women living in the community. ${ }^{11}$ Four hundred thirty-six women were enrolled at baseline and assessed at seven study visits from 1994 to 2008; 382 had information on $\mathrm{HbA} 1 \mathrm{c}$ levels available at baseline. After excluding participants with missing covariates $(n=11)$, stroke or Parkinson's disease (more likely to have lower extremity limitations due to primary disease; $\mathrm{n}=5$ ), or HbA1c levels less than $4.5 \% \quad(\mathrm{n}=2), 364$ women remained. The excluded women did not differ significantly from those included in the study.

For incident frailty analysis, women who had the outcome (frailty) at baseline $(n=11)$, missing outcome (frailty) status at baseline $(\mathrm{n}=2)$, or no follow-up $(\mathrm{n}=22)$ were also excluded, leaving 329 women available for this analysis. The total number of participants who completed study visits was as follows: two visits $(\mathrm{n}=25)$, three visits $(\mathrm{n}=58)$; four visits $(\mathrm{n}=22)$; five visits $(\mathrm{n}=26)$; six visits $(\mathrm{n}=74)$; and all seven visits $(\mathrm{n}=124)$.

Similar exclusion criteria were used for lower extremity outcomes, which resulted in the following analytical samples to examine incidence of self-reported walking difficulty $(\mathrm{n}=329)$; low walking speed, defined as a level in the lowest quartile $(<0.82 \mathrm{~m} / \mathrm{s})$ for the study population $(\mathrm{n}=259)$; and low physical performance, defined as a Short Physical Performance Battery (SPPB) score in the lowest quartile $(<9)$ for the study population $(\mathrm{n}=267)$.

\section{Variables}

The main outcome was frailty as described by Fried and colleagues. ${ }^{5,6}$ Five criteria were used: shrinking (body mass index (BMI) $<18.5 \mathrm{~kg} / \mathrm{m}^{2}$ or $\geq 5 \%$ annual weight loss), weakness (low grip strength), poor endurance (exhaustion), slowness (low walking speed), and physical inactivity. Those with no criteria were categorized as nonfrail, one or two criteria as prefrail, and three or more criteria as frail.

Lower extremity mobility outcomes included subjective and objective measures. Participants self-reported any difficulty walking one-quarter of a mile at all visits. Walking speed was calculated based on a usual-pace 4-m measured walk test at all visits. Assessment of the SPPB, consisting of chair stands, walk test, and tandem stands for balance, was available for all visits except visit 4 , and scores were calculated using criteria defined previously and adapted for clinical use. ${ }^{12-14}$

The main exposure of interest was HbA1c. Nonfasting blood samples were obtained and HbA1c measured using a BioRad assay (Hercules, CA) from frozen whole blood.9

Demographic information was obtained using a standardized questionnaire. Height and weight were measured to calculate BMI. BMI was categorized according to World Health Organization criteria as underweight $(<18.5 \mathrm{~kg} /$ $\left.\mathrm{m}^{2}\right)$, normal $\left(18.5-24.9 \mathrm{~kg} / \mathrm{m}^{2}\right)$, overweight $(25.0-29.9 \mathrm{~kg} /$ $\left.\mathrm{m}^{2}\right)$, and obese $\left(\geq 30 \mathrm{~kg} / \mathrm{m}^{2}\right){ }^{15}$ For the analysis of incident walking difficulty only, underweight was categorized as less than $20 \mathrm{~kg} / \mathrm{m}^{2}$, because all women with BMI less than $18.5 \mathrm{~kg} / \mathrm{m}^{2}$ developed walking difficulty. Interleukin (IL)-6 was measured from frozen serum using a commercial enzyme-linked immunosorbent assay (Quantikine Human; R\&D Systems, Minneapolis, MN). History of diabetes mellitus, coronary artery disease, osteoarthritis and chronic obstructive pulmonary disease was self-reported. Coronary artery disease included congestive heart failure, myocardial infarction, and angina pectoris.

Peripheral arterial disease was defined as an anklebrachial index less than $0.9 .^{3}$ Peripheral neuropathy was defined according to physician report or inability to feel the complete vibration of a $128 \mathrm{~Hz}$ tuning fork in either great toe.

\section{Statistical Analysis}

Baseline characteristics were compared according to incident frailty status using the chi-square test or Student $t$ test. Incidence was defined as first occurrence of each event in all analyses. Kaplan-Meier survival curves explored the association between HbA1c (categorized to account for potential nonlinear relationships into $<5.5 \%, 5.5-5.9 \%$, $6.0-6.4 \%, 6.5-7.9 \%, \geq 8.0 \%)$ and time to event for frailty and lower extremity outcomes. HbA1c categories were chosen based on previous studies suggesting greater risk of mortality at the highest and lowest thresholds, ${ }^{16,17}$ with cutoffs for the intermediate categories based on diagnostic criteria for diabetes mellitus. ${ }^{18}$ Cox regression models for discrete time outcomes were constructed to characterize the independent association between $\mathrm{HbA} 1 \mathrm{c}$ at baseline and outcomes in the following sequential models: Model 1: adjusted for demographics (age, race, 
education), Model 2: adjusted for Model 1 plus BMI, Model 3: adjusted for Model 2 plus IL-6, Model 4: adjusted for Model 3 plus comorbidities (coronary artery disease, osteoarthritis, chronic obstructive pulmonary disease, peripheral arterial disease, peripheral neuropathy). For the frailty model, sensitivity analyses were performed adjusting for prefrailty status at baseline. Potential quadratic associations between $\mathrm{HbA} 1 \mathrm{c}$ and incident frailty or other outcomes were explored in regression models, but because none were found, the lowest $\mathrm{HbA1c}$ category $(<5.5 \%)$ was chosen as the reference for all analyses. The statistical program used was SAS 9.2 version (SAS Institute, Inc., Cary, NC).

\section{RESULTS}

Seventy-seven of 329 women (23\%) developed incident frailty during a mean follow-up of $8.6 \pm 3.6$ years. Within the baseline nonfrail cohort, 70 women $(21.3 \%)$ died, and $57(17.4 \%)$ dropped out before frailty development. The women who did and did not develop frailty were similar with respect to age, race, and education (Table 1). A significant difference in the distribution of BMI categories was found between groups $(P=.01)$, with a greater proportion of women being obese or underweight in the group that developed frailty. Not surprisingly, a greater proportion of women were prefrail at baseline in the incident frailty group than in the nonfrail group $(55.8 \%$ vs $29.4 \% ; P<.001)$. There were no significant differences in the clinical history of other comorbidities (all $P>.05$ ). Inflammatory markers (IL-6) were largely similar, whereas mean $\mathrm{HbA1c}$ tended to be higher in women who developed incident frailty $(6.2 \%)$ than in those who did not $(6.0 \%, P=.07)$.

For lower extremity limitations, $27 \%$ of women developed self-reported walking difficulty (mean follow-up $8.4 \pm 3.7$ years), $63 \%$ of women developed low walking speed (mean follow-up $8.8 \pm 3.5$ years), and 67\% developed low physical performance (mean follow-up $8.8 \pm 3.5$ years). The overall incidence rate for frailty and lower extremity limitations during follow-up per 100 person-years was $2.26(95 \%$ confidence interval $(\mathrm{CI})=1.78$ 2.83) for frailty, $2.78(95 \% \mathrm{CI}=2.24-3.42)$ for walking difficulty, $8.46(95 \% \mathrm{CI}=7.20-9.88)$ for low walking speed, and $8.61(95 \% \mathrm{CI}=7.40-9.97)$ for low physical performance.

Kaplan-Meier survival curves were next examined for time-to-event analyses (Figure 1A-D ). The women were divided into the following HbA1c categories for incident frailty analysis: less than $5.5 \%(\mathrm{n}=64), 5.5 \%$ to $5.9 \%$ $(\mathrm{n}=135), \quad 6.0 \% \quad$ to $6.4 \% \quad(\mathrm{n}=77), 6.5 \%$ to $7.9 \%$ $(\mathrm{n}=38)$, and $8.0 \%$ or greater $(\mathrm{n}=15)$. Similar HbA1c categories were used for lower extremity outcomes. HbA1c category was associated with probability of developing frailty $(P=.10)$ and low walking speed $(P=.10)$, although the results were not statistically significant based on the log-rank test (Figure 1A, C). Nevertheless, HbA1c category was significantly associated with probability of developing incident walking difficulty (Figure $1 \mathrm{~B}, P=.049$ ) and low physical performance (Figure $1 \mathrm{D}, P=.001$ ).

The association between HbA1c category and incident frailty was further explored in regression models (Table 2).
Table 1. Selected Baseline Participant Characteristics According to Development of Frailty During Follow-Up

\begin{tabular}{|c|c|c|c|c|}
\hline \multirow[b]{2}{*}{ Characteristic } & \multirow[b]{2}{*}{$\begin{array}{c}\text { All, } \\
\mathrm{N}=329\end{array}$} & \multicolumn{3}{|c|}{$\begin{array}{c}\text { Incident Frailty During } \\
\text { Follow-Up }\end{array}$} \\
\hline & & $\begin{array}{c}\text { Yes, } \\
\mathrm{n}=77\end{array}$ & $\begin{array}{c}\text { No, } \\
n=252\end{array}$ & $\begin{array}{c}P \text { - } \\
\text { value }\end{array}$ \\
\hline \multicolumn{5}{|l|}{ Demographic } \\
\hline Age, mean \pm SD & $73.9 \pm 2.8$ & $74.0 \pm 2.9$ & $73.9 \pm 2.8$ & .62 \\
\hline White, \% & 83.9 & 83.1 & 84.1 & .83 \\
\hline $\begin{array}{l}\text { Education, years, } \\
\text { mean } \pm \text { SD }\end{array}$ & $12.6 \pm 3.3$ & $12.2 \pm 2.9$ & $12.8 \pm 3.4$ & .17 \\
\hline \multicolumn{5}{|c|}{ Body mass index, $\mathrm{kg} / \mathrm{m}^{2}(\%)$} \\
\hline$<18.5$ & 3.3 & 6.5 & 2.4 & .01 \\
\hline $18.5-24.9$ & 36.2 & 29.9 & 38.1 & \\
\hline $25.0-29.9$ & 38.9 & 31.2 & 41.3 & \\
\hline$\geq 30.0$ & 21.6 & 32.5 & 18.3 & \\
\hline Mean \pm SD & $26.6 \pm 5.1$ & $27.3 \pm 5.5$ & $26.4 \pm 4.9$ & .16 \\
\hline \multicolumn{5}{|l|}{ Clinical history, $\%$} \\
\hline Prefrailty & 35.6 & 55.8 & 29.4 & $<.001$ \\
\hline Osteoarthritis & 66.9 & 66.2 & 67.1 & .89 \\
\hline $\begin{array}{l}\text { Chronic obstructive } \\
\text { pulmonary disease }\end{array}$ & 23.4 & 26.0 & 22.6 & .54 \\
\hline $\begin{array}{l}\text { Coronary artery } \\
\text { disease }\end{array}$ & 13.7 & 16.9 & 12.7 & .35 \\
\hline $\begin{array}{l}\text { Peripheral arterial } \\
\text { disease }\end{array}$ & 4.6 & 7.8 & 3.6 & .12 \\
\hline $\begin{array}{l}\text { Peripheral } \\
\text { neuropathy }\end{array}$ & 7.0 & 7.8 & 6.8 & .76 \\
\hline $\begin{array}{l}\text { Known diabetes } \\
\text { mellitus }\end{array}$ & 7.6 & 9.1 & 7.1 & .57 \\
\hline \multicolumn{5}{|l|}{ Laboratory measure } \\
\hline $\begin{array}{l}\text { Interleukin-6, pg/ } \\
\mathrm{mL} \text {, mean } \pm \mathrm{SD}\end{array}$ & $3.9 \pm 4.8$ & $4.2 \pm 7.0$ & $3.8 \pm 3.9$ & .49 \\
\hline \multicolumn{5}{|c|}{ Glycosylated hemoglobin (\%) } \\
\hline$<5.5 \%$ & 19.5 & 15.6 & 20.6 & .24 \\
\hline $5.5-5.9 \%$ & 41.0 & 40.3 & 41.3 & \\
\hline $6.0-6.4 \%$ & 23.4 & 24.7 & 23.0 & \\
\hline $6.5-7.9 \%$ & 11.6 & 10.4 & 11.9 & \\
\hline$\geq 8.0 \%$ & 4.5 & 9.1 & 3.2 & \\
\hline $\bar{M}$ ean \pm SD & $6.0 \pm 0.9$ & $6.2 \pm 1.1$ & $6.0 \pm 0.8$ & .07 \\
\hline
\end{tabular}

$\mathrm{BMI}=$ body mass index; $\mathrm{SD}=$ standard deviation.

${ }^{\text {a }}$ Comparing participants with incident frailty to those without incident frailty.

HbA1c of $8.0 \%$ or greater was significantly associated with incident frailty after adjusting for demographic characteristics (hazard ratio $(\mathrm{HR})=3.63,95 \% \quad \mathrm{CI}=$ 1.41-9.33; Model 1) compared to the reference category $(\mathrm{HbA} 1 \mathrm{c}<5.5 \%)$. This association was slightly attenuated after further adjustment for BMI and IL- 6 but essentially unchanged. After adjustment for comorbidities, the association remained significant $(\mathrm{HR}=3.33,95 \% \mathrm{CI}=1.24$ 8.93; Model 4). In sensitivity analyses, prefrailty was further adjusted for in the fully adjusted model to explore its contributions as a potential confounder; ${ }^{9}$ the association was attenuated such that it was no longer significant $(\mathrm{HR}=2.64,95 \% \mathrm{CI}=0.95-7.34)$. However, prefrailty may also contribute as a mediator of the association between $\mathrm{HbA} 1 \mathrm{c}$ and incident frailty in women who are nonfrail at baseline.

HbA1c of $8.0 \%$ or greater was also associated with significantly more difficulty in walking after adjustment for 

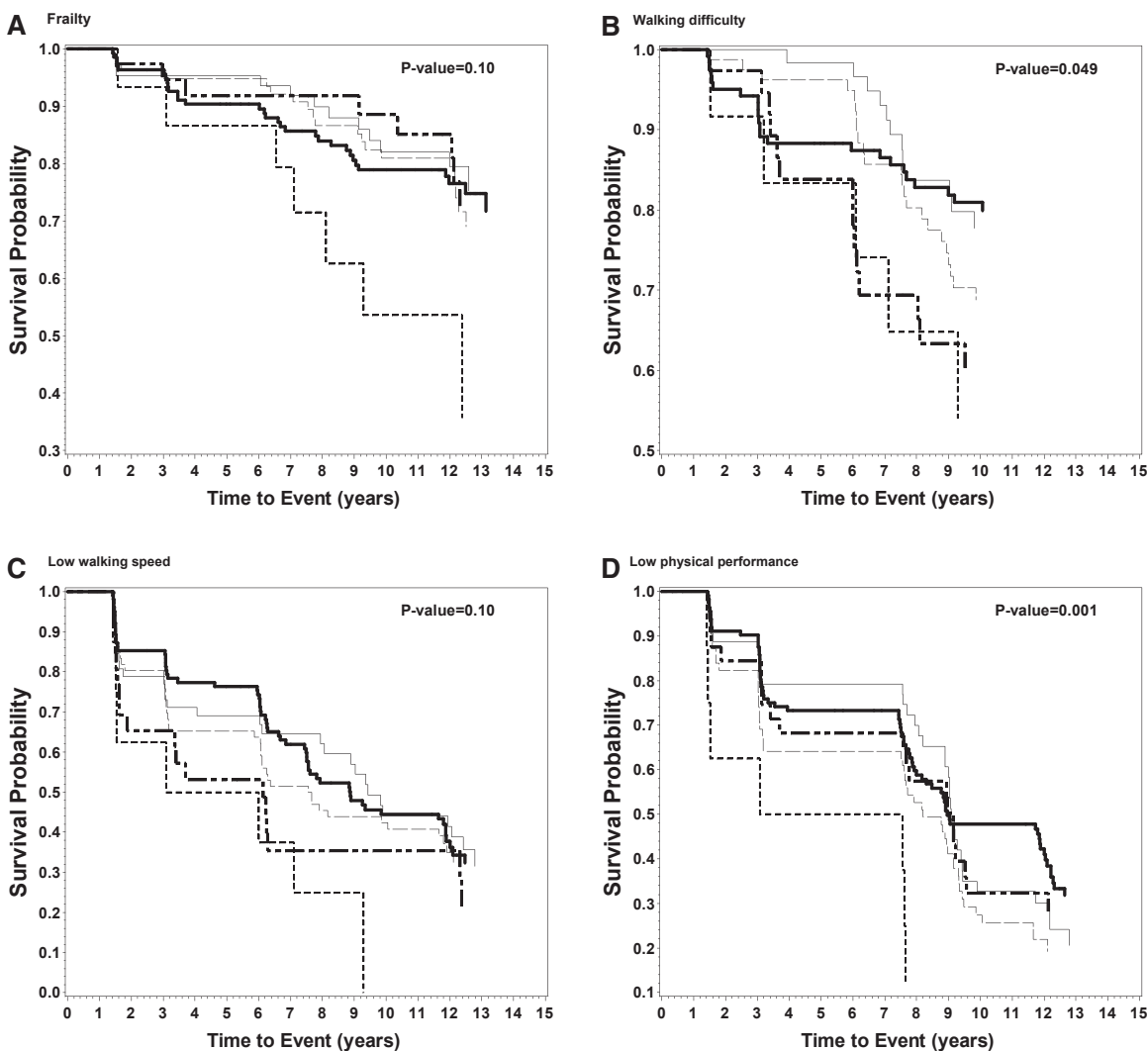

Figure 1. Kaplan-Meier curves demonstrating the time to event of outcomes during follow-up for older women categorized according to level of baseline glycosylated hemoglobin (HbA1c): $<5.5 \%$ (thin solid line —-), 5.5-5.9\% (thick solid line - -), 6.0-6.4\% (thin dashed line - -), 6.5-7.9\% (thick short and long dashed line - - -), and $\geq 8 \%$ (thick short dashed line --- ). The association between HbA1c category and probability of survival for the outcomes are $(\mathrm{A})$ frailty $(P=.10)$; $(\mathrm{B})$ self-reported walking difficulty $(P=.049)$; $(\mathrm{C})$ slow walking speed $(P=.10)$; and (D) low Short Physical Performance Battery score $(P=.001)$. The $x$-axis shows the time to event in years. The $y$-axis shows the probability of survival.

demographic characteristics $(\mathrm{HR}=5.03,95 \% \mathrm{CI}=1.91$ 13.27; Model 1) compared to the reference category (Table 2). This association was moderately attenuated but remained significant after adjustment for BMI $(\mathrm{HR}=2.93$, 95\% CI $=1.08-7.96 ;$ Model 2$), \mathrm{IL}-6 \quad(\mathrm{HR}=2.91,95 \%$ $\mathrm{CI}=1.07-7.91 ;$ Model 3), and comorbidities $(\mathrm{HR}=3.47$, $95 \%$ CI $=1.26-9.55$; Model 4 ). HbA1c of $6.5 \%$ to $7.9 \%$ was also significantly associated with incident walking difficulty in models adjusted for demographic characteristics $(\mathrm{HR}=2.26,95 \% \mathrm{CI}=1.05-4.87$; Model 1$)$, suggesting a possible graded association between $\mathrm{HbA} 1 \mathrm{c}$ level and incident walking difficulty, although this was no longer statistically significant in fully adjusted models (Model 4).

Similarly, HbA1c level of $8.0 \%$ or greater was associated with development of low walking speed after adjustment for demographic characteristics ( $\mathrm{HR}=3.14,95 \%$ $\mathrm{CI}=1.35-7.34 ;$ Model 1$), \mathrm{BMI} \quad(\mathrm{HR}=2.89,95 \% \mathrm{CI}=$ 1.23-6.81; Model 2), IL-6 (HR $=2.70,95 \%$ CI $=1.15-$ 6.33; Model 3), and comorbidities $(\mathrm{HR}=2.82,95 \% \mathrm{CI}=$ 1.19-6.71) compared to HbA1c $<5.5 \%$.

Last, HbA1c of $8.0 \%$ or greater (versus reference) was associated with development of poor physical performance after adjustment for demographic characteristics $(\mathrm{HR}=3.31,95 \% \mathrm{CI}=1.43-7.69), \mathrm{BMI}(\mathrm{HR}=3.25,95 \%$ $\mathrm{CI}=1.38-7.66), \mathrm{IL}-6(\mathrm{HR}=3.09,95 \% \mathrm{CI}=1.31-7.28)$, and comorbidities $(\mathrm{HR}=3.60,95 \% \mathrm{CI}=1.52-8.53)$.

\section{DISCUSSION}

Participants in the highest HbA1c category $(\geq 8.0 \%)$ had a statistically significantly three times greater risk of developing frailty and three to five times greater risk of developing lower extremity mobility limitations after adjustment for demographics than those in the lowest category $(<5.5 \%)$. The association between HbA1c and incident frailty and lower extremity mobility limitations remained independent of potential confounders and was nonlinear, with most events occurring in the highest $\mathrm{HbA1c}$ category $(\geq 8.0 \%)$, suggesting that hyperglycemia, particularly in the diabetic range, can predict the onset of incident frailty and lower extremity mobility limitations less than a decade later.

To the knowledge of the authors, the association between hyperglycemia and incident frailty has been explored in only one other study. ${ }^{11}$ In that study, homeostasis model of insulin resistance (HOMA-IR) was calculated based on fasting glucose and insulin. For every standard deviation increment in HOMA-IR, the adjusted HR for frailty was $1.15(95 \% \mathrm{CI}=1.02-1.31)$. In comparison, the current study found that the association between $\mathrm{HbA} 1 \mathrm{c}$ and incident frailty was nonlinear. An advantage of $\mathrm{HbA1c}$ is that postprandial hyperglycemia, which may be more related to frailty status than fasting 
Table 2. Frailty and Lower Extremity Mobility Limitations in the Women's Health and Aging Study II, According to Glycosylated Hemoglobin at Baseline

\begin{tabular}{|c|c|c|c|c|}
\hline \multirow[b]{2}{*}{ Glycosylated Hemoglobin Level,\% } & \multicolumn{4}{|c|}{ Adjusted Hazard Ratio (95\% Confidence Interval) } \\
\hline & Model 1 & Model 2 & Model 3 & Model 4 \\
\hline $5.5-5.9$ & $1.28(0.65-2.49)$ & $1.35(0.69-2.65)$ & $1.30(0.66-2.55)$ & $1.29(0.65-2.55)$ \\
\hline $6.0-6.4$ & $1.18(0.57-2.45)$ & $1.26(0.59-2.67)$ & $1.25(0.59-2.65)$ & $1.25(0.58-2.69)$ \\
\hline $6.5-7.9$ & $1.17(0.44-2.81)$ & $1.05(0.41-2.7)$ & $1.01(0.39-2.61)$ & $1.04(0.40-2.70)$ \\
\hline $5.5-5.9$ & $1.10(0.59-2.05)$ & $1.18(0.63-2.21)$ & $1.17(0.62-2.20)$ & $1.14(0.60-2.15)$ \\
\hline $6.0-6.4$ & $1.36(0.71-2.61)$ & $1.25(0.64-2.43)$ & $1.24(0.64-2.41)$ & $1.28(0.65-2.51)$ \\
\hline $6.5-7.9$ & $2.26(1.05-4.87)$ & $1.70(0.77-3.75)$ & $1.70(0.77-3.75)$ & $1.77(0.79-3.97)$ \\
\hline$\geq 8.0$ & $5.03(1.91-13.27)$ & $2.93(1.08-7.96)$ & $2.91(1.07-7.91)$ & $3.47(1.26-9.55)$ \\
\hline \multicolumn{5}{|l|}{ Low walking speed ${ }^{a}$} \\
\hline $5.5-5.9$ & $1.04(0.67-1.61)$ & $1.06(0.68-1.66)$ & $1.04(0.67-1.63)$ & $1.02(0.65-1.59)$ \\
\hline $5.5-5.9$ & $0.99(0.66-1.50)$ & $1.01(0.67-1.54)$ & $1.00(0.66-1.53)$ & $0.97(0.63-1.49)$ \\
\hline $6.0-6.4$ & $1.23(0.78-1.93)$ & $1.24(0.79-1.96)$ & $1.19(0.75-1.89)$ & $1.19(0.75-1.88)$ \\
\hline $6.5-7.9$ & $1.14(0.64-2.02)$ & $1.13(0.63-2.02)$ & $1.05(0.58-1.88)$ & $1.06(0.58-1.92)$ \\
\hline$\geq 8.0$ & $3.31(1.43-7.69)$ & $3.25(1.38-7.66)$ & $3.09(1.31-7.28)$ & $3.60(1.52-8.53)$ \\
\hline
\end{tabular}

Model 1 adjusted for age, race, education. Model 2 adjusted for variables in Model 1 and body mass index. Model 3 adjusted for variables in Model 2 and interleukin-6. Model 4 adjusted for variables in Model 3 and clinical history of comorbidities (osteoarthritis, chronic obstructive pulmonary disease, coronary artery disease, peripheral arterial disease, and peripheral neuropathy).

${ }^{a}$ Walking speed in the lowest quartile $(<0.82 \mathrm{~m} / \mathrm{s})$.

${ }^{\mathrm{b}}$ Short Performance Physical Battery score in the lowest quartile $(<9)$.

levels alone, also influences levels. ${ }^{8}$ The other advantages of $\mathrm{HbA1c}$ are that it can be obtained without fasting and may be less variable with repeat testing. ${ }^{19}$ Thus, the results of the current study provide a new perspective on a previously described relationship between hyperglycemia and incident frailty.

It has been demonstrated that higher HbA1c levels are cross-sectionally associated with walking difficulties. ${ }^{9}$ The present study reports for the first time that the development of difficulty in self-reported walking or performancebased measures of lower extremity function is greater in persons with $\mathrm{HbA} 1 \mathrm{c}$ levels of $8.0 \%$ or greater than in those with levels less than $5.5 \%$ at baseline, independent of confounders. Objective measures may also detect preclinical limitations that predict future disability. ${ }^{12,14}$

Evidence was not found of a J-shaped association between $\mathrm{HbA} 1 \mathrm{c}$ and incident frailty or lower extremity outcomes, although such associations have been described between HbA1c and mortality. ${ }^{16,17,20}$ Explanations for this discordance include the potential relationship between hypoglycemia and sudden death ${ }^{21}$ unrelated to the presence of frailty. Furthermore, frailty events occurring right before death may have been undetected in the analyses, yet when the composite outcome of incident frailty or death was examined, the results were unchanged (data not shown), although smaller numbers of participants with high HbA1c levels in the current study may have limited the ability to detect quadratic associations.

The association between hyperglycemia and incident frailty may be due to several factors. Similar to other studies, the current study found that frail women were more likely to be obese, which may be associated with chronic inflammation. ${ }^{22}$ Inflammation is further associated with lower leg muscle mass and strength, which in turn is related to functional impairment, physical disability, and frailty. ${ }^{12,23,24}$ Lower muscle function is inherent in the definition of frailty. In addition, chronic hyperglycemia is a risk factor for cardiovascular disease, which in turn has been associated with frailty, ${ }^{25}$ although the current study found that hyperglycemia was related to incident frailty status independent of the potential contributions of adiposity, inflammation, and cardiovascular disease. It also found that participants with hyperglycemia were more likely to develop lower extremity mobility limitations independent of confounders and that associations were independent of potential mediators such as peripheral arterial disease and peripheral neuropathy. ${ }^{3}$

A possible implication of these findings is that direct pathways linking hyperglycemia to muscle loss need to be considered. Insulin resistance and diabetes mellitus have been associated with excessive loss of lean body mass and muscle strength in observational studies. ${ }^{26,27}$ Insulin resistance is also associated with skeletal muscle mitochondrial dysfunction, ${ }^{28}$ which may suggest underlying pathways for these epidemiological findings, but further studies are needed.

Limitations of this study include the small sample size of participants in the highest HbA1c category $(\geq 8.0 \%)$, although it was possible to discern significant associations between $\mathrm{HbA1c}$ and incident frailty and lower extremity mobility limitations in adjusted regression models. The study included only women, limiting generalizability. There was also high mortality, probably because of the old 
age of the women, although these occurred nonsystematically (data not shown). Only baseline HbA1c levels were explored, although changes in HbA1c levels over time may contribute to frailty status as well. Furthermore, the majority of persons with $\mathrm{HbA} 1 \mathrm{c}$ of $8.0 \%$ or greater had a known history of diabetes mellitus, so it was not possible to separate the effect of $\mathrm{HbA} 1 \mathrm{c}$ from the presence of diabetes mellitus itself. As a result, the possibility that other aspects of the diabetic state, such as the use of glucoselowering therapies, could contribute to frailty or that higher HbA1c levels in older persons reflects poorer selfcare management and greater risk of adverse outcomes cannot be excluded. Lastly, although the study focused on an individual measure (HbA1c), recent studies have suggested that deficits across multiple systems may be most important in frailty development. ${ }^{29}$ It is likely that complex interactions between dysglycemia and abnormalities in other physiological systems contribute to the pathophysiology of frailty, which it is hoped will be explored in a future study. Strengths of the current study include the well-characterized Women's Health and Aging Study II cohort, use of standardized protocols, inclusion of selfreported and performance-based measures of lower extremity function, examination of potential nonlinear relationships, and length of follow-up. Furthermore, use of a long-term glycemic marker (HbA1c) minimized potential variability in exposure.

In conclusion, this study adds to growing evidence that hyperglycemia is independently associated with the development of frailty and with incident lower extremity mobility limitations. HbA1c testing may represent a practical method to screen individuals at high risk for the development of adverse geriatric outcomes, but whether this greater risk of adverse geriatric outcomes is primarily related to HbA1c levels in the moderately uncontrolled diabetic range or higher $(\geq 8.0 \%)$ needs to be further explored. A better understanding might provide insight into whether clinical guidelines proposing less-aggressive HbA1c targets in older adults with diabetes mellitus are appropriate. ${ }^{30}$ Although the mechanism remains unclear, direct associations between hyperglycemia and muscle loss may contribute and should be investigated in future studies. Intervention studies are ultimately needed to explore whether treatment of hyperglycemia may delay or prevent the development of frailty and lower extremity limitations in older adults.

\section{ACKNOWLEDGMENTS}

Conflict of Interest: This work was supported by the Johns Hopkins Older Americans Independence Center (P30-AG021334), National Institute on Aging (R37AG19905 and R01AG021493), and the Veterans Affairs Ann Arbor Geriatrics Research, Education and Clinical Center.

Author Contributions: Kalyani, Xue, Walston, Cappola, Fried, Brancati, and Blaum: Study concept and design, methods, analysis and interpretation of data, and preparation of manuscript. Cappola, Blaum, Fried, and Walston: Data acquisition. Tian: Study methods, data analysis, and interpretation of data.

Sponsor's Role: None.

\section{REFERENCES}

1. Cowie CC, Rust KF, Ford ES et al. Full accounting of diabetes and pre-diabetes in the U.S. population in 1988-1994 and 2005-2006. Diabetes Care 2009;32:287-294.

2. Wild S, Roglic G, Green A et al. Global prevalence of diabetes: Estimates for the year 2000 and projections for 2030. Diabetes Care 2004;27:10471053.

3. Volpato S, Ferrucci L, Blaum C et al. Progression of lower-extremity disability in older women with diabetes: The Women's Health and Aging Study. Diabetes Care 2003;26:70-75.

4. Kalyani RR, Saudek CD, Brancati FL, Selvin E. The association of diabetes, comorbidities, and hemoglobin A1c with functional disability in older adults: Results from the National Health and Nutrition Examination Survey (NHANES), 1999-2006. Diabetes Care 2010;33:1055-1060.

5. Fried LP, Tangen CM, Walston J et al. Frailty in older adults: Evidence for a phenotype. J Gerontol A Biol Sci Med Sci 2001;56A:M146-M156.

6. Bandeen-Roche K, Xue QL, Ferrucci L et al. Phenotype of frailty: Characterization in the Women's Health and Aging Studies. J Gerontol A Biol Sci Med Sci 2006;61A:262-266.

7. Walston J, McBurnie MA, Newman A et al. Frailty and activation of the inflammation and coagulation systems with and without clinical comorbidities. Arch Intern Med 2002;162:2333-2341.

8. Kalyani RR, Varadhan R, Weiss CO et al. Frailty status and altered glucose-insulin dynamics. J Gerontol A Biol Sci Med Sci 2011. [Epub ahead of print]

9. Blaum CS, Xue QI, Tian J et al. Is hyperglycemia associated with frailty status in older women? J Am Geriatr Soc 2009;57:840-847.

10. Barzilay JI, Blaum C, Moore $\mathrm{T}$ et al. Insulin resistance and inflammation as precursors of frailty: The Cardiovascular Health Study. Arch Intern Med 2007;167:635-641.

11. Fried LP, Bandeen-Roche K, Chaves PH et al. Preclinical mobility disability predicts incident mobility disability in older women. J Gerontol A Biol Sci Med Sci 2000;55A:M43-M52.

12. Guralnik JM, Simonsick EM, Ferrucci L et al. A short physical performance battery assessing lower extremity function: Association with selfreported disability and prediction of mortality and nursing home admission. J Gerontol 1994;49:M85-M94.

13. Guralnik JM, Ferrucci L, Pieper CF et al. Lower extremity function and subsequent disability: Consistency across studies, predictive models, and value of gait speed alone compared with the Short Physical Performance Battery. J Gerontol A Biol Sci Med Sci 2000;55A:M221-M231.

14. Studenski S, Perera S, Wallace D et al. Physical performance measures in the clinical setting. J Am Geriatr Soc 2003;51:314-322.

15. Obesity: Preventing and Managing the Global Epidemic. Report of a WHO Consultation on Obesity. Geneva, Switzerland: World Health Organization, 2000 .

16. Selvin E, Steffes MW, Zhu H et al. Glycated hemoglobin, diabetes, and cardiovascular risk in nondiabetic adults. N Engl J Med 2010;362:800 811.

17. Huang ES, Liu JY, Moffet HH et al. Glycemic control, complications, and death in older diabetic patients: The Diabetes and Aging Study. Diabetes Care 2011;34:1329-1336.

18. American Diabetes Association. Standards of medical care in diabetes2011. Diabetes Care 2011;34:S11-S61.

19. Selvin E, Crainiceanu CM, Brancati FL et al. Short-term variability in measures of glycemia and implications for the classification of diabetes. Arch Intern Med 2007;167:1545-1551.

20. Action to Control Cardiovascular Risk in Diabetes Study Group, Gerstein $\mathrm{HC}$, Miller ME et al. Effects of intensive glucose lowering in type 2 diabetes. N Engl J Med 2008;358:2545-2559.

21. Frier BM, Schernthaner G, Heller SR. Hypoglycemia and cardiovascular risks. Diabetes Care 2011;34:S132-S137.

22. Hubbard RE, Lang IA, Llewellyn DJ et al. Frailty, body mass index, and abdominal obesity in older people. J Gerontol A Biol Sci Med Sci 2010;65 A:377-381.

23. Visser M, Kritchevsky SB, Goodpaster BH et al. Leg muscle mass and composition in relation to lower extremity performance in men and women aged 70 to 79: The Health, Aging and Body Composition Study. J Am Geriatr Soc 2002;50:897-904.

24. Ferrucci L, Penninx BW, Volpato S et al. Change in muscle strength explains accelerated decline of physical function in older women with high interleukin-6 serum levels. J Am Geriatr Soc 2002;50:1947-1954.

25. Newman AB, Gottdiener JS, Mcburnie MA et al. Associations of subclinical cardiovascular disease with frailty. J Gerontol A Biol Sci Med Sci 2001;56A:M158-M166. 
26. Park SW, Goodpaster BH, Lee JS et al. Excessive loss of skeletal muscle mass in older adults with type 2 diabetes. Diabetes Care 2009;32:19931997.

27. Park SW, Goodpaster BH, Strotmeyer ES et al. Accelerated loss of skeletal muscle strength in older adults with type 2 diabetes: The Health, Aging, and Body Composition Study. Diabetes Care 2007;30:1507-1512.

28. Phielix E, Schrauwen-Hinderling VB, Mensink M et al. Lower intrinsic ADP-stimulated mitochondrial respiration underlies in vivo mitochondrial dysfunction in muscle of male type 2 diabetic patients. Diabetes 2008;57:2943-2949.

29. Fried LP, Xue QL, Cappola AR et al. Nonlinear multisystem physiological dysregulation associated with frailty in older women: Implications for etiology and treatment. J Gerontol A Biol Sci Med Sci 2009;64A:1049-1057.

30. Brown AF, Mangione CM, Saliba D et al. Guidelines for improving the care of the older person with diabetes mellitus. J Am Geriatr Soc 2003;51: S265-S280. 